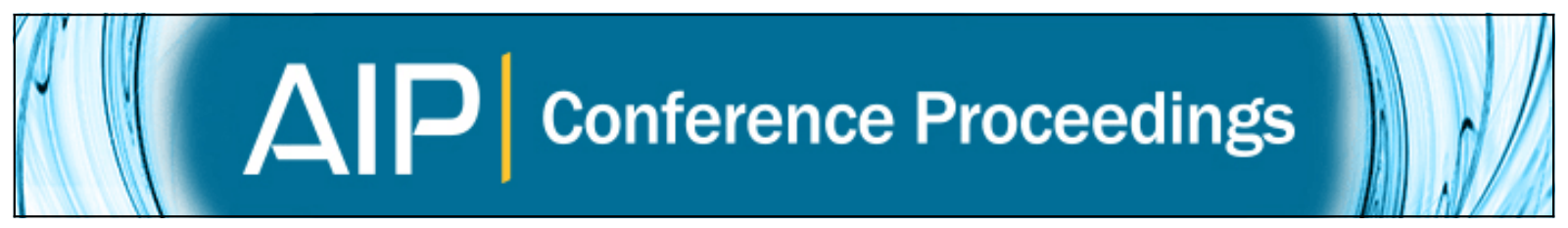

\title{
Bulk and Surface Charge Collection: CDMS Detector Performance and Design Implications
}

C. N. Bailey, Z. Ahmed, D. S. Akerib, S. Arrenberg, D. Balakishiyeva, L. Baudis, D. A. Bauer, J. Beaty, P. L. Brink , T. Bruch, R. Bunker, B. Cabrera, D. O. Caldwell, K. Clark, J. Cooley, P. Cushman, F. DeJongh, M. R.

Dragowsky, L. Duong, E. Figueroa-Feliciano, J. Filippini, M. Fritts, S. R. Golwala, D. R. Grant, J. Hall, R. Hennings-Yeomans, S. Hertel, A. Hojem, D. Homgren, L. Hsu, M. E. Huber, O. Kamaev, M. Kiveni, M. Kos, S. W. Leman, R. Mahapatra, V. Mandic, D. Moore, K. A. McCarthy, N. Mirabolfathi, H. Nelson, R. W. Ogburn, M. Pyle, X. Qiu, E. Ramberg, W. Rau, A. Reisetter, T. Saab, B. Sadoulet, J. Sander, R. W. Schnee, D. N. Seitz, B. Serfass, K. M. Sundqvist, A. Tomada, G. Wang, M. Whilden, S. Yellin, J. Yoo, and B. A. Young

Citation: AIP Conference Proceedings 1185, 643 (2009); doi: 10.1063/1.3292423

View online: http://dx.doi.org/10.1063/1.3292423

View Table of Contents: http://scitation.aip.org/content/aip/proceeding/aipcp/1185?ver=pdfcov

Published by the AIP Publishing

Articles you may be interested in

Characterization of SuperCDMS 1-inch Ge Detectors

AIP Conf. Proc. 1185, 659 (2009); 10.1063/1.3292428

SuperCDMS Detector Fabrication Advances

AIP Conf. Proc. 1185, 655 (2009); 10.1063/1.3292427

Composite CaWO 4 Detectors for the CRESST-II Experiment

AIP Conf. Proc. 1185, 651 (2009); 10.1063/1.3292426

The Cryogenic Dark Matter Search (CDMS) experiment: Results, status and perspective AIP Conf. Proc. 1185, 623 (2009); 10.1063/1.3292418

Charge and heat collection in a $70 \mathrm{~g}$ heat/ionization cryogenic detector for dark matter search J. Appl. Phys. 87, 1507 (2000); 10.1063/1.372042 


\title{
Bulk and Surface Charge Collection: CDMS Detector Performance and Design Implications
}

\author{
C.N. Bailey ${ }^{\mathrm{a}}$, Z. Ahmed ${ }^{\mathrm{b}}$, D.S. Akerib ${ }^{\mathrm{a}}$, S. Arrenberg ${ }^{\mathrm{c}}$, D. Balakishiyeva ${ }^{\mathrm{d}}$,
} L. Baudis ${ }^{\mathrm{c}}$, D.A. Bauer ${ }^{\mathrm{e}}$, J. Beaty ${ }^{\mathrm{f}}$, P.L. Brink ${ }^{\mathrm{g}}$, T. Bruch ${ }^{\mathrm{c}}$, R. Bunker ${ }^{\mathrm{h}}$, B. Cabrera ${ }^{\mathrm{g}}$, D.O. Caldwell ${ }^{\mathrm{h}}$, K. Clark ${ }^{\mathrm{a}}$, J. Cooley ${ }^{\mathrm{g}}$, P. Cushman ${ }^{\mathrm{f}}$, F. DeJongh ${ }^{\mathrm{e}}$,

M.R. Dragowsky ${ }^{\mathrm{a}}$, L. Duong ${ }^{\mathrm{f}}$, E. Figueroa-Feliciano ${ }^{\mathrm{i}}$, J. Filippini ${ }^{\mathrm{j}, \mathrm{b}}$, M. Fritts ${ }^{\mathrm{f}}$, S.R. Golwala ${ }^{b}$, D.R. Grant ${ }^{\mathrm{a}}$, J. Hall ${ }^{\mathrm{e}}$, R. Hennings-Yeomans ${ }^{\mathrm{a}}$, S. Hertel ${ }^{\mathrm{i}}$, A. Hojem ${ }^{\mathrm{a}}$, D. Homgren ${ }^{\mathrm{e}}$, L. Hsu ${ }^{\mathrm{e}}$, M.E. Huber ${ }^{\mathrm{k}}$, O. Kamaev ${ }^{\mathrm{f}}$, M. Kiveni ${ }^{1}$, M. Kos ${ }^{1}$, S.W. Leman ${ }^{\mathrm{i}}$, R. Mahapatram ${ }^{\mathrm{m}}$, V. Mandic ${ }^{\mathrm{f}}$, D. Moore ${ }^{\mathrm{b}}$, K.A. McCarthy ${ }^{\mathrm{i}}$,

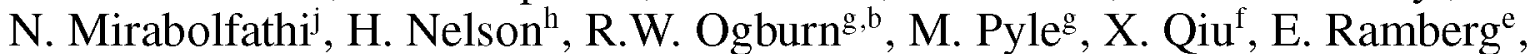
W. Rau ${ }^{\mathrm{n}}$, A. Reisetter ${ }^{\mathrm{o}, \mathrm{f}}$, T. Saab ${ }^{\mathrm{d}}$, B. Sadoulet ${ }^{\mathrm{p}, \mathrm{j}}$, J. Sander $^{\mathrm{h}}$, R.W. Schnee ${ }^{\mathrm{g}}$, D.N. Seitz ${ }^{j}$, B. Serfass ${ }^{j}$, K.M. Sundqvist ${ }^{j}$, A. Tomada ${ }^{\mathrm{g}}$, G. Wang ${ }^{\mathrm{b}}$, M. Whilden ${ }^{\mathrm{a}}$, S. Yellin ${ }^{\mathrm{g}, \mathrm{h}}, \mathrm{J}$. Yoo ${ }^{\mathrm{e}}$ and B.A. Young ${ }^{\mathrm{q}}$

\author{
${ }^{a}$ Department of Physics, Case Western Reserve University, Cleveland, OH 44106, USA \\ ${ }^{b}$ Department of Physics, California Institute of Technology, Pasadena, CA 91125, USA \\ ${ }^{c}$ Physics Institute, University of Zurich, Zurich, Switzerland \\ ${ }^{d}$ Department of Physics, University of Florida, Gainesville, FL 32611, USA \\ ${ }^{e}$ Fermi National Accelerator Laboratory, Batavia, IL 60510, USA \\ ${ }^{f}$ School of Physics \& Astronomy, University of Minnesota, Minneapolis, MN 55455, USA \\ ${ }^{g}$ Department of Physics, Stanford University, Stanford, CA 94305, USA \\ ${ }^{h}$ Department of Physics, University of California, Santa Barbara, CA 93106, USA \\ ${ }^{i}$ Department of Physics, Massachusetts Institute of Technology, Cambridge, MA 02139, USA \\ ${ }^{j}$ Department of Physics, University of California, Berkeley, CA 94720, USA \\ ${ }^{k}$ Departments of Phys. \& Engr., University of Colorado Denver, Denver, CO 80217, USA \\ ${ }^{l}$ Department of Physics, Syracuse University, Syracuse, NY 13244, USA \\ ${ }^{m}$ Department of Physics, Texas A\&M University, College Station, TX 93106, USA \\ ${ }^{n}$ Department of Physics, Queen's University, Kingston, ON, Canada, K7L $3 N 6$ \\ ${ }^{\circ}$ Department of Physics, Saint Olaf College, Northfield, MN 55057, USA \\ ${ }^{p}$ Lawrence Berkeley National Laboratory, Berkeley, CA 94720, USA \\ ${ }^{q}$ Department of Physics, Santa Clara University, Santa Clara, CA 95053, USA
}

\begin{abstract}
The Cryogenic Dark Matter Search (CDMS) searches for Weakly Interacting Massive Particles (WIMPs) with cryogenic germanium particle detectors. These detectors discriminate between nuclear-recoil candidate and electron-recoil background events by collecting both phonon and ionization energy from interactions in the crystal. Incomplete ionization collection results in the largest background in the CDMS detectors as this causes electron-recoil background interactions to appear as false candidate events. Two primary causes of incomplete ionization collection are suface and bulk charge trapping. Recent work has been focused on reducing surface trapping through the modification of fabrication methods for future detectors. Analyzing data taken with test devices shows that hydrogen passivation of the amorphous silicon blocking layer does not reduce the effects of surface trapping. Other data shows that the iron-ion implantation used to lower the critical temperature of the tungsten transition-edge sensors increases surface trapping, causing a degradation of the ionization collection. Using selective implantation on future detectors may improve ionization collection for events near the phonon side detector surface. Bulk trapping is minimized by neutralizing ionized lattice impurities. Detector investigations at testing facilities and at the experimental site in Soudan, $\mathrm{MN}$ have provided methods to optimize the neutralization process and monitor running conditions to maintain maximal ionization collection.
\end{abstract}

Keywords: CDMS, SuperCDMS, Dark Matter, Charge Collection

PACS: 95.35.+d, 14.80.Ly, 73.61.Jc, 74.78.-w, 85.25.Oj, 85.25.-j

CP1185, Low Temperature Detectors LTD 13, Proceedings of the $13^{\text {th }}$ International Workshop

edited by B. Cabrera, A. Miller, and B. Young

(C) 2009 American Institute of Physics 978-0-7354-0751-0/09/\$25.00 


\section{INTRODUCTION}

The Cryogenic Dark Matter Search (CDMS) [1] experiment is located at the Soudan Underground Laboratory and uses Z-sensitive Ionization and Phonon (ZIP) detectors to measure both phonon and ionization energy, with phonon sensors on one flat detector face and ionization sensors on the other. This dual measurement technique allows event-by-event discrimination between electronrecoil background events, arising from residual radioactivity and cosmic rays, and nuclear-recoil signal events, possibly produced by Weakly Interacting Massive Particles (WIMPs). CDMS's primary discrimination parameter is ionization yield, the ratio of ionization to phonon recoil energy. Electron-recoil events produce a higher ionization yield than nuclear-recoil events, allowing discrimination of nuclear from electron recoils with a rejection factor of $>10^{4}$. Ideally, ionization yield would provide complete discrimination of electron recoils. However, one of the most significant sources of background in CDMS detectors is due to charge trapping. This trapping can occur through several mechanisms, either in the bulk or at the surface of the crystal. In both cases charge trapping reduces the total ionization signal, causing electron recoils to occur at lower ionization yield, thereby mimicing nuclear recoils. Currently CDMS minimizes these effects through the use of phonon timing information to reject surface events and neutralization to minimize bulk trapping. To increase sensitivity to WIMP interactions, the next phase of CDMS, SuperCDMS [2], is increasing the target mass. Reducing charge trapping can improve background rejection and help keep the experiment background free at larger masses.

\section{SURFACE TRAPPING}

Surface trapping creates a "dead layer," which is characterized by interactions with incomplete charge collection. This surface trapping is caused by the backdiffusion of charge carriers into the nearby ionization electrode. To separate and drift charge carriers to the electrodes, CDMS detectors are biased with a small electric field $(\sim 3 \mathrm{~V} / \mathrm{cm})$. If an interaction occurs near the detector surface the electric field is not strong enough to separate all the charge carriers before some reach the adjacent ionization electrode. Since these carriers do not drift across the crystal they do not contribute to the ionization signal (which is proportional to the carrier drift length), therefore causing a reduction in the measured ionization energy.

Both the phonon and ionization detector sides are patterned with a tungsten and aluminum grid to bias the detector. Additional Al fins feed into $\mathrm{W}$ transitionedge sensors for phonon signal collection [1]. Adding amorphous silicon between the detector crystal and $\mathrm{Al}$ layer was found to reduce the size of the dead layer $[3,4]$. While this solution reduces the dead layer, electron recoils occuring in the first $\sim 5-10 \mu \mathrm{m}$ (depending on detector side and applied electric field) of the detector surfaces still suffer low ionization yield that, in extreme cases, can mimic the bulk nuclear-recoil signal. These surface electron recoils are rejected by taking advantage of phonon timing information.

Calibration studies of a Ge ZIP detector found that the dead layer on the phonon side of the detector is larger than that on the detector's ionization side [5] while subsequent performance studies of Soudan data show that the ionization yield based rejection factor for surface events occurring on the phonon side is $\sim 4$ times worse than for ionization side surface events in Ge detectors [6]. Recently two hypotheses have been tested to decrease the phonon side dead layer and remove this asymmetry between phonon and ionization side surface events.

\section{Hydrogenated Amorphous Silicon}

One approach to reducing the size of the phonon side dead layer was to passivate the amorphous silicon blocking layer with hydrogen. Previous experience with hydrogenated amorphous silicon (HaSi) blocking layers in early CDMS detectors $[3,4]$ indicates an improvement in ionization yield based surface event discrimination. This improvement is thought to be due to the passivation of interface defects in the amorphous silicon (aSi) [7, 8], therefore increasing the layer's blocking ability and decreasing the detector dead layer.

While initial tests hydrogenating the aSi layer in 1inch silicon detectors resulted in promising results, that under specific bias conditions the asymmetry would disappear [9], the technique needed to be tested with germanium detectors (since most CDMS detectors are made from $\mathrm{Ge}$ rather than $\mathrm{Si}$ ). Three Ge test devices were fabricated: one "control" device with no hydrogen added to the aSi layer on either detector face (like CDMS-II detectors), one device with $20 \%$ hydrogen added to the aSi layer on the ionization face, and the final device with $8 \%$ hydrogen added to the aSi layer on the phonon face (Table 1). By mapping the collected ionization signal for $60 \mathrm{keV}$ events (from collimated ${ }^{241} \mathrm{Am}$ sources) with the expected depth distribution (based on the penetration depth for $60 \mathrm{keV}$ events), the phonon and ionization side dead layers were determined for the three Ge test devices. The dead layer is defined to be the depth with 63\% ionization collection and has negligible statistical errors. Systematic errors were determined by examining dead layer variations for the same detector during different runs and multiple source locations during the same 
TABLE 1. Detectors used to study how adding hydrogen to the aSi blocking layer affects ionization collection.

\begin{tabular}{|c|c|c|c|c|c|}
\hline Detector & Thickness & Phonon Side Surface & Charge Side Surface & Ge Doping Type & Impurity Concentration \\
\hline G3D & 1 inch & $\mathrm{aSi}$ & $\mathrm{aSi}$ & p-type & $7.7 \times 10^{+10}$ to $7.6 \times 10^{+10}$ \\
\hline G2E & 1 inch & $\mathrm{aSi}$ & $20 \% \mathrm{HaSi}$ & n-type & $1.5 \times 10^{+10}$ to $8.0 \times 10^{+10}$ \\
\hline G28B & $1 \mathrm{~cm}$ & $8 \% \mathrm{HaSi}$ & $\mathrm{aSi}$ & n-type & $2.7 \times 10^{+11}$ to $4.7 \times 10^{+11}$ \\
\hline
\end{tabular}

run. This shows typical variations up to $\sim 30 \%$, with a few outliers as high as $60 \%$. Based on this analysis, the detector with no hydrogenation (G3D) showed the best dead layer performance [6], (Figure 1). This suggests that adding hydrogen to the aSi layer does not improve surface event ionization collection. This result is consistent with theories stating that, unlike on Si substrates, hydrogen added to a Ge crystal does not passivate interfacial dangling-bond defects $[10,11]$. However, this data showed detector-to-detector dead layer variations that may overwhelm a small effect due to the addition of hydrogen into the amorphous silicon layer. While this study was as controlled as possible, the three detectors analyzed did have differences in doping type (p-type vs. n-type), impurity concentrations, and fabrication batches which certainly could also have contributed to the observed dead layer variations.

\section{Iron-Ion Implantation}

Another possible way to solve the ionization yield discrimination asymmetry between the phonon and ionization side surface events is to determine its underlying cause and fix that. One hypothesis for the asymmetry is that it is due to the iron-ion implantation used on the phonon detector side to create appropriate and uniform tungsten superconducting transition temperatures [12]. The iron-ion implantation is optimized so that the peak of the iron-ion concentration is approximately $40 \%$ through the $\mathrm{W}$ layer [13]. However, the majority of the incident iron-ion flux strikes portions of the detector surface not covered by $\mathrm{W}$. These parasitic ions are suspected to cause damage to the Ge surface by adding acceptors and thus increasing the dead layer.

To test this hypothesis, one of the test Ge devices (G3D) had half of its ionization side iron-ion implanted for comparison between the implanted and nonimplanted ionization side dead layers. Like the analysis studying the effects of hydrogenation in the aSi layer, the size of the ionization side dead layer was determined for the implanted and non-implanted detector halves using data from $60 \mathrm{keV}$ calibration gammas. This analysis shows that the dead layer for the implanted half was systematically larger than the non-implanted half [6], (Figure 1). Thus, iron-ion implantation may indeed be one of

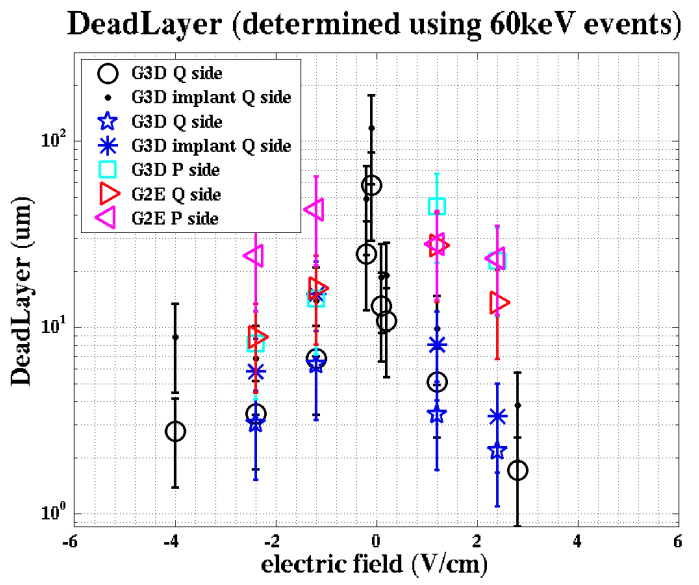

FIGURE 1. Dead layer as a function of applied electric field comparing G3D with G2E, demonstrating how G3D (no hydrogen in the aSi layer on either detector face) has the smallest dead layer for a given applied electric field. Comparison of the ionization side dead layers of G3D (black circle and blue star) and G2E (red triangle) illustrate how hydrogenating the ionization side aSi layer doesn't improve the dead layer while comparison of the phonon side dead layers of G3D (cyan square) and G2E (magenta triangle) show detector-to-detector variations between two detector faces that are nominally the same. Comparison of G3D's non-implanted (black circle and blue star) and implanted (black dot and blue closed star) ionization sides show how iron-ion implantation creates a larger dead layer. Black and blue points represent data taken with G3D, only during separate data runs. Error bars on the data points represent $50 \%$ variations to the determined dead layer. Based on the systematic error analysis, these error bars represent maximal variations.

the causes of the ionization yield asymmetry for phonon and ionization side surface events. However, comparing the ionization and phonon side dead layers indicates that this may not be the only cause of the asymmetry. In the future, selective masking may be used during implantation to ensure that only the $\mathrm{W}$ on each detector sees the iron-ion flux. It is also important to note that devices fabricated recently, using a new $\mathrm{W}$ sputtering target, require $75 \%$ less ion dosage to suppress the $\mathrm{T}_{c}$ to the optimum values. Thus, any ill effects implantation may have on charge collection may automatically be reduced to a truly insignificant level. 


\section{BULK TRAPPING}

Bulk trapping reduces the ionization signal by trapping charge carriers at impurities or crystal lattice defects. To reduce the impact of bulk trapping, CDMS detector crystals have low impurity $\left(<1 \mathrm{e}^{11} / \mathrm{cm}^{3}\right)$ and dislocation $\left(<5000 / \mathrm{cm}^{2}\right)$ concentrations. Operationally, data taking occurs on time scales shorter than the time when bulk trapping affects ionization collection. Additionally, detectors routinely undergo a neutralization process to reduce the effects of bulk trapping. During this in situ neutralization process detectors are grounded while excess electrons and holes are generated using either an external gamma source of LEDs. These free charge carriers execute a random walk through the crystal undergoing Coulomb attraction with ionized impurity sites which neutralize these sites. This minimizes bulk trapping because neutral impurity sites have lower trapping cross sections than ionized sites [1].

During the Soudan 5-tower runs [14], there was substantial effort to determine a quantitative method for monitoring the neutralization states of the detectors. Evaluating the fraction of low yield events (the ratio of the number of events below the electron-recoil band to the total number of events) has proven to be a powerful method for establishing and comparing detector neutralization states. In particular, this quantitative figure-ofmerit of detector neutralization allowed the development of a new data quality cut [6] which removed any data sets that potentially suffered from poor detector neutralization, like the candidate event from the Soudan 2-tower data analysis [15], and real-time data monitoring to ensure stable detector neutralization. Finally, having this figure-of-merit allowed investigations of detector neutralization at testing facilities and in situ at the experimental site which have provided methods to optimize the neutralization process. These studies have shown that a combination of neutralization techniques (using LEDs and strong radioactive sources) produces the best detector neutralization. In addition these studies have been used to modify the LED parameters so that they produce effective neutralization while minimizing the heat load to the cryostat and shown that LEDs, mounted in the detector housing, neutralize both the detector it is mounted to as well as the adjacent detector.

\section{CONCLUSION}

The first 5-tower data runs of CDMS-II has resulted in a world leading WIMP-nucleon spin-independent cross section limit for WIMP masses above $44 \mathrm{GeV} / \mathrm{c}^{2}$ [14]. As CDMS aims to achieve greater WIMP sensitivity, detector modifications have already been made to increase the target mass and reduce backgrounds $[2,16]$. The stud- ies discussed here, to reduce the detector dead layer, will impact further modifications to SuperCDMS detectors by implementing the use of selective iron-ion implantation which will reduce the phonon side dead layer. Neutralization studies have already led to improved background rejection, real-time monitoring (for better data quality), and optimizations to neutralization procedures. Further work at understanding charge transport in low temperature detector crystals [17] will lead to further operational optimizations to maintain full ionization collection.

\section{ACKNOWLEDGMENTS}

This work is supported in part by the National Science Foundation (Grant Nos. AST-9978911, PHY-0542066, PHY-0503729, PHY-0503629, PHY-0503641, PHY0504224, PHY-0705052, PHY-0801536, PHY-0801708, PHY-0801712 and PHY-0802575), by the Department of Energy (Contracts DE-AC03-76SF00098, DE-FG02-91ER40688, DE-FG02-92ER40701, DEFG03-90ER40569, and DE-FG03-91ER40618), by the Swiss National Foundation (SNF Grant No. 20-118119), and by NSERC Canada (Grant SAPIN 341314-07).

\section{REFERENCES}

1. D. Akerib et al., Phys Rev D 72 (2005).

2. D. Akerib et al., Low Temp Phys 151, 818-823 (2008).

3. T. Shutt et al., Nucl Instrum Methods A 444, 340-344 (2000).

4. T. Shutt et al., Low Temp Det (2002).

5. V. Mandic et al., Nucl Instrum Methods A 520, 171-174 (2004).

6. C. Bailey, The Cryogenic Dark Matter Search: First 5-Tower Data and Improved Understanding of Ionization Collection, Ph.D. thesis, Case Western Reserve University (2009).

7. A. Stesmans, Appl Phys Lett 68 (1996).

8. C. Van de Walle, and R. Street, Phys Rev B 49 (1994).

9. C. Bailey et al., Low Temp Phys 151, 211-215 (2008).

10. J. Weber, A. Janotti, P. Rinke, and C. Van de Walle, Appl Phys Lett 91 (2007).

11. C. Van de Walle, and J. Neugebauer, Nature 423 (2003).

12. B. Young et al., Nucl Instrum Methods A 444, 296-299 (2000).

13. B. Young et al., Appl Phys 86 (1999).

14. Z. Ahmed et al., Phys Rev Lett 102 (2009).

15. D. Akerib et al., Phys Rev Lett 96 (2006).

16. Z. Ahmed et al., "Characterization of SuperCDMS 1-inch Ge Detectors," in the se proceedings, 2009.

17. K. Sundqvist, and B. Sadoulet, Low Temp Phys 151, 443-447 (2008). 